\title{
Effect of Muscle Fatigue on the Proprioception by the Taekwondo Training Type
}

\author{
Byoung-Do Seo $\cdot$ Hui-Jae $\mathrm{Kim}^{1 \dagger} \cdot$ Joung-Youl Ju \\ Kyungwoon University, Department of Physical Therapy, \\ ${ }^{1}$ Kyungsung University, Department of Physical Therapy
}

Received: April 20, 2020 / Revised: April 29, 2020 / Accepted: July 1, 2020

(C) 2020 J Korean Soc Phys Med

\section{| Abstract |}

PURPOSE: This study examined the effects of muscle fatigue on proprioception in the type of taekwondo training, causing injury to taekwondo athletes.

METHODS: The subjects were divided into Taekwondo Kyorugi athletes $(\mathrm{n}=14)$ and Taekwondo Poomsae athletes $(\mathrm{n}=14)$. After inducing muscle fatigue to the subjects, the joint position sense (JPS) and the force reproduction sense (FRS) were measured before and immediately after the induction of muscle fatigue.

RESULTS: Studies have shown no significant impact on the JPS and FRS caused by muscle fatigue induced by Poomsae and Kyorugi ( $p>$.05). The JPS, however, showed a significant difference before and after Kyorugi $(\mathrm{p}<.05)$. The FRS showed significant differences before and after Poomsae $(\mathrm{p}<.05)$. Through this study, the injuries to taekwondo athletes appear to be influenced by the joint position and force

This paper is a summary of Kim Hui Jae (2017)'s master's degree thesis.

$\dagger$ Corresponding Author : Hui-Jae Kim

physiotherapist@kakao.com, https://orcid.org/0000-0002-7713-1725 This is an Open Access article distributed under the terms of the Creative Commons Attribution Non-Commercial License (http://creativecommons.org/licenses/by-nc/3.0) which permits unrestricted non-commercial use, distribution, and reproduction in any medium, provided the original work is properly cited. reproduction. Moreover, the injury appears to affect the joint position sense in Kyorugi athletes and the force reproduction sense in Poomsae athletes.

CONCLUSION: The results will contribute to injury prevention and athlete protection. In addition, the information can be used as basic data for the development of sports injuries prevention and rehabilitation programs for Taekwondo athletes. Research is needed on the customized management of sports methods and self-care to prevent sports damage, considering the athletic characteristics of the taekwondo athletes in the future.

Key Words: Sports injury, Proprioceptive sense, Joint position sensation, Force reproduction sense

\section{Introduction}

Taekwondo is a sport that requires an athlete to exert explosive force in a short time. The sport can cause a range of injuries to the lower extremities, reducing the athlete's performance. In extreme cases, an injured athlete may have to give up his/her career as a professional athlete. Therefore, for proper injury prevention, Taekwondo athletes and athlete trainers must understand the causes of injuries to the lower limbs in Taekwondo athletes [1].

Taekwondo athletes are exposed to the risk of sports injuries to the lower limbs during training and competition 
[2]. This is because Taekwondo competition involves the continuous and instantaneous exertion of explosive power during three rounds of a three-minute match, which leads to muscle fatigue. The kinematic characteristics of Taekwondo include rapid kicking and torso rotation, which are influenced by the dynamic and static balance abilities of the body [3]. Muscle fatigue is defined as a condition in which the capacity of each muscle fiber to contract is reduced, and results from excessive physical activities or repetitive weight-bearing exercise [4]. Muscle fatigue can result in a variety of sports injuries, including contact or non-contact anterior cruciate ligament injuries in competitive sports, such as Taekwondo, soccer, and hockey, or sports that involve jumping and landing, such as volleyball and basketball.

In Taekwondo athletes, muscle fatigue causes muscle weakness and changes in proprioception, resulting in decreased agility and speed as well as momentary losses of balance ability. These conditions may cause sports injuries to the leg supporting the body during one-leg kicking or retracting of the kicking leg to its original position because of an insufficient position sense. Therefore, research on proprioception of Taekwondo athletes can contribute to a basic understanding of sports injuries in Taekwondo athletes and benefit intervention efforts for sports injury prevention through proprioception enhancement training [5].

Physical therapists or athlete trainers often prescribe proprioception enhancement exercises to athletes because proprioception plays a vital role in reducing the functional instability and re-injury to the joints [6,7]. These exercises improve the balance ability and postural control, thereby reducing the risks of injuries to the knee or ankle joints during exercise [8]. In addition, proprioception enhancement exercise is recommended as an essential component of rehabilitation exercise because it helps athletes quickly rehabilitate and prevent recurrence after injuries to the knee and ankle joints when it is combined with musclestrengthening exercise [9]. Given that related studies are consistently reporting that proprioception has direct and indirect effects on sports injuries, quantitative and qualitative studies on proprioception are needed to determine the causes of sports injuries to knee and ankle joints, which frequently occur in Taekwondo athletes.

Taekwondo competitions are composed mainly of Kyorugi (full-contact sparring) and Poomsae competitions [3]. The muscle used, type of muscle fatigue, and changes in the proprioception before and after muscle fatigue will differ according to the form of competition an athlete is performing [10]. This is because Kyorugi athletes are optimally trained in exerting power and fast torso rotation to strike the opponent. In contrast, Poomsae athletes characteristically demonstrate static and dynamic movements in a non-competitive manner, focusing on the temperate and accurate performance of a series of actions from the beginning to the end of Poomsae [11]. Kyorugi athletes mainly engage in movements with strong dynamic kinematic characteristics, such as quick kicking and retracting of the kicking leg to its original position. On the other hand, Poomsae athletes mainly engage in movements with static kinematic characteristics, such as maintaining the extended leg to show the correct posture [12]. Therefore, the type of muscle fatigue and the changes in proprioception before and after muscle fatigue should differ between these two groups.

Therefore, in reference to the factors that induce noncontact sports injuries in Taekwondo athletes, this study examined the effects of muscle fatigue on proprioception according to the type of Taekwondo training, and to provide basic data on sports injuries in Taekwondo athletes.

\section{Methods}

\section{Study Design and Period}

This study was designed as a pre-post test and control group study on 28 Taekwondo athletes of $\mathrm{K}$ University from November 15 to 20, 2017 (Five days). 


\section{Study Subjects}

The Taekwondo athletes of $\mathrm{K}$ university participating in this study were divided into two groups: Kyorugi athlete group $(n=14)$ and Poomsae athlete group $(n=14)$. After a thorough explanation of the purpose of this study to potential research participants, oral consent was received from those who voluntarily decided to participate in this study. The research participants had to meet the following criteria: Taekwondo athletes with no neurological disease, metabolic disease, or vascular disease that could affect the balance ability and had not undergone any surgery for knee joint problems within the last six months.

\section{Experimental Procedures}

To measure changes in proprioception due to muscle fatigue, the joint position sense (JPS) and force reproduction sense (FRS) were measured before and after inducing muscle fatigue in both groups. The evaluation was conducted at the Sports Science Laboratory at K University. The laboratory was kept at $22{ }^{\circ} \mathrm{C}$ and $60 \%$ humidity to minimize changes in the physiological responses due to environmental factors. The participants were asked to wear a lightweight short-sleeve T-shirt and shorts.

\section{1) Induce of Muscle Fatigue}

According to a study on the effects of muscle fatigue, recovery from induced muscle fatigue on ankle joints takes place within 10 to 30 minutes after induced fatigue, albeit with differences according to the exercise intensity and the properties of the induced fatigue [14]. In the case of knee joints, it was reported that recovery from the fatigue effect induced by a five-second maximal isometric voluntary contraction occurs within the first one to two minutes after the exercise [15]. In this study, the landing motion was restricted to 10 minutes after muscle fatigue was induced by the fatigue-inducing exercise, and the measurements were taken as quickly as possible to prevent a decrease in fatigue effect [10].

\section{2) Knee Joint Position Sense (JPS) Assessment}

An assessment of the knee joint position sense (JPS) was performed on a table in the sitting position using a $360^{\circ}$ joint range-of-motion measuring device for the lower limbs (Baseline Metal Goniometer-360 Degree Range-14 Inch Leg, JAMAR, USA) [16]. The starting position was defined as $0^{\circ}$ (knee flexed at $90^{\circ}$ ), and the target angle was set to $50^{\circ}$ (knee flexed at $40^{\circ}$ ). After blindfolding the subject, the researcher extended the subject's knee joint on the dominant leg to the target angle and sustained the target angle position for five seconds, allowing the subject to remember that position. The subject was then asked to reproduce the joint position according to memory. After sustaining the target-joint position for five seconds, the subject's knee joint was returned passively to the starting position. Fifteen seconds later, the subject was asked to extend the knee joint voluntarily on the dominant leg to the target angle. Before taking the measurements, a maker was attached to the lateral malleolus and the fibular head of the subject's dominant leg. The joint position reproduced by the subject was photographed using a digital camera (Mirrorless NX-2000, Samsung, Korea), and the 2D motion analysis program Twinner Pro (SIMI reality motion systems $\mathrm{GmbH}$, Germany) was used to take measurements to two decimal places [17]. Measurements were taken for flexion and extension. The measured value was defined as the error value between the angle suggested by the researcher and the angle performed actively by the subject (unit: ${ }^{\circ}$ ). Measurements were taken three times to calculate the mean error. A smaller error indicates a more accurate JPS.

\section{3) Force Reproduction Sense (FRS) Assessment}

The force reproduction sense (FRS) was assessed in the sitting position on a chair with a back to prevent errors caused by the movement of the trunk [18]. The subject was instructed to sit on the chair with the trunk touching the back of the chair at all times. A scale tilted at $10^{\circ}$ (HE-70 Digital Scale, CAS, Korea) was placed on the floor. 
Table 1. General Characteristics of the Subjects $(N=28)$

\begin{tabular}{ccccc}
\hline Variable & Kyorugi Group $(\mathrm{n}=14)$ & Poomsae Group $(\mathrm{n}=14)$ & t-value & $\mathrm{p}$-value \\
\hline Height $(\mathrm{cm})$ & $172.50 \pm 4.38$ & $171.46 \pm 6.59$ & .482 & .629 \\
Weight $(\mathrm{kg})$ & $65.10 \pm 6.45$ & $72.90 \pm 14.13$ & -.081 & .937 \\
Thigh circumference $(\mathrm{cm})$ & $49.14 \pm 3.38$ & $46.03 \pm 3.38$ & 1.806 & .083 \\
\hline
\end{tabular}

Mean \pm SD

The subject's entire sole, except for the heels, was placed on the scale to measure the force reproduction (Unit: $\mathrm{kg}$ ). The subject was asked to press the scale to reach the target force and maintain it for three seconds using only the ankle plantar flexor force. The target force was set to $40 \%$ of the subjects' average maximum force calculated in kilograms. After setting the target force, the subject was asked to practice producing the target force three times while using the sight to perceive all the necessary visual input. For the actual assessment, the subject was blindfolded and instructed to reproduce the target force. Measurements of the force reproduction were taken three times; before, immediately after and 10 minutes after the muscle fatigue was induced.

\section{Statistical Analyses}

The results were analyzed using the SPSS for Windows20.0 program. The general characteristics of the participants were determined through descriptive statistics to yield the mean and standard deviation. Normality was found through a Shapiro-Wilk test. The general characteristics and the test of homogeneity of the pre-intervention values for each group were found through an independent t-test. An independent t-test was performed to compare the results between the groups. A paired t-test was used to compare the dependent variables within the groups. The statistically significant level was set to $a=.05$.

\section{Results}

\section{General Characteristics of the Subjects}

Table 1 lists the general characteristics of the subjects. In the Kyorugi group, the mean height, weight, and thigh circumference of the dominant leg were $172.50 \pm 4.38$ $\mathrm{cm}, 65.10 \pm 6.45 \mathrm{~kg}$, and $49.14 \pm 3.38 \mathrm{~cm}$, respectively. In the Poomsae group, the mean height, weight, and thigh circumference of the dominant leg were $171.46 \pm 6.59$ $\mathrm{cm}, 72.90 \pm 14.13 \mathrm{~kg}$, and $46.03 \pm 3.38 \mathrm{~cm}$, respectively. Based on a comparison of the homogeneity between the two groups, the significance probability indicated no difference between the two groups $(p>.05)$.

\section{Comparison of the Changes in the JPS}

According to the assessment results of the JPS for extension, the mean error of the Kyorugi group before muscle fatigue and 10 minutes after muscle fatigue was $-20 \pm 3.27^{\circ}$ and $3.86 \pm 3.18^{\circ}$, respectively. The mean error of the Poomsae group before muscle fatigue and 10 minutes after muscle fatigue was $-29 \pm 2.67^{\circ}$ and $1.45 \pm 5.92^{\circ}$, respectively. In the Kyorugi group, the mean error before and after muscle fatigue showed a significant difference $(p<.05)$. In the Poomsae group, the mean error before and after muscle fatigue showed no significant difference $(\mathrm{p}>.05)$. Moreover, there was no significant difference between the groups $(\mathrm{p}>.05)$ (Table 2).

According to the assessment results of the JPS for flexion, the mean error of the Kyorugi group before muscle fatigue and 10 minutes after muscle fatigue was $-2.07 \pm$ $3.12^{\circ}$ and $-1.43 \pm 6.50^{\circ}$, respectively. The mean error of 
Table 2. Assessment Results of Proprioception $(\mathrm{N}=28)$

\begin{tabular}{|c|c|c|c|c|c|}
\hline variable & & Kyorugi Group $($ Mean \pm SD) & Poomsae Group (Mean \pm SD) & t-value & p-value \\
\hline \multirow{4}{*}{ JPS (Extension) $\left(^{\circ}\right)$} & Pre & $-.20 \pm 3.27$ & $-.29 \pm 2.67$ & \multirow{2}{*}{1.353} & \multirow{2}{*}{.188} \\
\hline & Post & $3.86 \pm 3.18$ & $1.45 \pm 5.92$ & & \\
\hline & t-value & -4.80 & -1.06 & & \\
\hline & $\mathrm{p}$-value & $.000^{* * *}$ & .306 & & \\
\hline \multirow{4}{*}{ JPS (Flexion) $\left(^{\circ}\right)$} & Pre & $-2.07 \pm 3.12$ & $-1.42 \pm 2.44$ & \multirow{2}{*}{.724} & \multirow{2}{*}{.465} \\
\hline & Post & $-1.43 \pm 6.50$ & $-3.07 \pm 5.14$ & & \\
\hline & t-value & -.391 & 1.223 & & \\
\hline & p-value & .700 & .2421 & & \\
\hline \multirow{4}{*}{ FRS $(\mathrm{kg})$} & Pre & $3.91 \pm 1.26$ & $3.56 \pm 1.15$ & \multirow{2}{*}{3.940} & \multirow{2}{*}{.587} \\
\hline & Post & $3.35 \pm .46$ & $3.51 \pm 1.14$ & & \\
\hline & t-value & 1.850 & -2.983 & & \\
\hline & $\mathrm{p}$-value & .087 & $.011^{* *}$ & & \\
\hline
\end{tabular}

${ }^{*} \mathrm{p}<.05,{ }^{* *} \mathrm{p}<.01,{ }^{* * *} \mathrm{p}<.00$. JPS: Joint position sense, FRS: Force reproduction sense

the Poomsae group before muscle fatigue and 10 minutes after muscle fatigue was $-1.42 \pm 2.44^{\circ}$ and $-3.07 \pm 5.14^{\circ}$, respectively. In each group, the mean errors before and after muscle fatigue were similar $(\mathrm{p}>.05)$. Furthermore, there was no significant difference between the groups ( $p$ $>.05)$.

\section{Comparison of the Changes in the FRS}

According to the assessment results of the FRS, the mean force reproduced by the Kyorugi group before muscle fatigue and 10 minutes after muscle fatigue was $3.91 \pm$ $1.26 \mathrm{~kg}$ and $3.35 \pm .46 \mathrm{~kg}$, respectively. The mean force reproduced by the Poomsae group before muscle fatigue and 10 minutes after muscle fatigue was $3.56 \pm 1.15$ and $3.51 \pm 1.14$, respectively. In the Kyorugi group, the mean errors before and after muscle fatigue were similar $(\mathrm{p}>$. 05). In the Poomsae group, the mean error before and after muscle fatigue showed a significant difference $(p<.05)$. Furthermore, there was no significant difference between the groups $(\mathrm{p}>.05)$.

\section{Discussion}

The study results showed that muscle fatigue affects the JPS and FRS in Taekwondo athletes. Significant differences in the JPS of the extensor muscle after muscle fatigue were observed in the Kyorugi group, whereas significant differences in the FRS after muscle fatigue were observed in the Poomsae group. Regarding the characteristic changes in the JPS, the extensor muscles showed a negative mean error value before muscle fatigue but a positive mean error value after induced muscle fatigue. On the other hand, the flexor muscles showed negative mean errors both before and after muscle fatigue, recovering after 10 minutes after muscle fatigue was induced. In other words, muscle fatigue leads to a decrease in the knee joint angle during knee joint flexion but a further increase in joint angle during knee joint extension. Moreover, the recovery of the JPS was faster for knee joint extensor muscles than the flexor muscles.

Choi, Kim, and Hong [6] assessed the knee JPS before and after muscle fatigue with 15 men and 15 women in their 20 s and reported a significant difference in the error 
angle of the knee joint reposition between before and after muscle fatigue. Khil and Son [11] examined the knee JPS in 150 healthy women using a goniometer. They reported that the error angles of the knee joint position increase significantly with the older age group and lower exercise frequency. The results of the present study correspond to the findings of these previous studies, confirming that muscle fatigue affects the JPS.

Regarding the FRS, there was no significant difference between the groups. On the other hand, significant differences were observed depending on the timing of the measurement. The error range measured immediately after the induced muscle fatigue was larger than that before muscle fatigue, suggesting that the FRS becomes less accurate with muscle fatigue. Muscle fatigue generally reduces the muscle strength and leads to a decrease in the muscle response speed and recovery ability, insufficient proprioception, and decreased balance ability [19]. A previous study examined the FRS of the elbow joint flexor muscles before and after muscle fatigue with adult males [20]. Their subjects perceived $20 \%$ of their maximal voluntary force and measured their force reproduction for three seconds after induced muscle fatigue. The study results showed that the reproduction error significantly increased after induced muscle fatigue. Their findings correspond to the results of the present study, confirming that muscle fatigue affects the FRS.

These findings suggest that induced muscle fatigue affects the JPS and FRS in Taekwondo athletes. In addition, the characteristic changes in the JPS during knee joint flexion and extension suggest that muscle fatigue can lead to sense errors between the joints, between body segments and between body and space in Taekwondo athletes. These proprioception errors can become factors of sports injuries resulting from a decreased ability to adjust for the accurate distance during training or competition, resulting in reduced precision in striking capabilities, reduced balancing with one leg during kicking attacks, and inaccurate motion when retracting the kicking leg to its original position. The direct mechanism of proprioception affecting sports injuries in Taekwondo athletes that corroborates this interpretation has not been identified. Nevertheless, the findings of this study and the implications they have for Taekwondo athletes can be explained indirectly by the results reported by Kim and Youm [10]. They investigated the effects of knee joint muscle fatigue on the factors related to the mechanism resulting in lower limb injuries during landing, such as the time required and angular displacement and moments of the lower limb joints.

According to Kim and Youm [10], the angular displacement of the ankle joints decreased significantly in the coronal and horizontal planes after fatigue. Regarding the ankle joint moments, the plantar flexor moment decreased significantly in the sagittal plane after fatigue. As for the knee joint moments, the extensor moment significantly decreased in the sagittal plane after fatigue. Regarding the hip joint moments, the extensor moment showed a significant increase in the coronal plane after fatigue. These results suggest that the knee joint muscle group fatigue during landing increases the dynamic stability by minimizing the movements of the ankle, knee, and hip joints in the coronal and the horizontal planes and maintaining a neutral position as a complementary movement to protect the knee. Therefore, accumulated fatigue of the knee joint muscles may be strongly correlated with the increased incidence of injuries during landing after jumping, descending stairs, and downhill walking. Thus, these findings and interpretation can explain the kinematic changes and sports injury mechanism due to degraded proprioception in Taekwondo athletes.

Shin and Youm [21] examined the effects of ankle joint muscle fatigue on postural control during a single-leg stance to provide a more direct explanation of the effects of muscle fatigue that may occur during a single-leg stance associated with one-leg kicking movement in Taekwondo athletes. According to the study results, single-leg stance postural 
control decreased during the fatigue inducement method of plantar and dorsiflexion at an angular velocity of $30 \%$ in both the anteroposterior and mediolateral factors. This suggests that during high-intensity, short-duration exercise involving plantar and dorsiflexion at an angular velocity of $30 \%$, and fatigue at $50 \%$ of the maximum plantarflexion torque might result in reduced single-leg stance postural control. High-intensity and short-duration exercise with the plantar and dorsiflexion fatigue protocol at an angular velocity of $30 \%$ may be a determining factor for postural control impairment during a single-leg stance compared to long-duration, low-intensity exercise with the fatigue protocol at an angular velocity of $120 \%$ s. In addition, according to the studies by Adlerton, Moritz, and Moe-Nilssen [14] and Seo, Shin and Han [22], who reported that the effects of muscle fatigue on the femoral hamstringsto-quadriceps (H:Q) ratio changes at the landing time during plyometric exercise. In addition, muscle fatigue might cause damage to the anterior cruciate ligament and posterior cruciate ligament of the knee joint at the time of landing through a decrease or increase in the $\mathrm{H} / \mathrm{Q}$ ratio. This shows indirectly that the degraded proprioceptive sense between body segments due to muscle fatigue can lead to sports injuries in Taekwondo athletes.

In this study, it was hypothesized that the measurement variables would differ between the Kyorugi group and the Poomsae group. On the other hand, the results showed no significant difference between the groups in all the measured variables. Kim, Kim, and Shin [23] investigated the effects of isokinetic fatigue induced to the dominant ankle on static and dynamic postural control with six Kyorugi athletes and 22 Poomsae athletes. They found that the isokinetic muscle function of the dominant ankle was significantly higher in the Poomsae group at plantar flexion peak torque, indicating a difference between the groups. Cha and Oh[24] also showed that the muscle strength at an angular velocity of $60 \% \mathrm{sec}$ in the hip isokinetic extensor and flexor was significantly higher in the left extensor among the National Taekwondo demonstration player group than the Kyorugi group $(\mathrm{p}<.05)$. They also reported that, when measured at an angular velocity of $180^{\circ} / \mathrm{sec}$, the muscle strength in the right extensor was significantly higher in the Taekwondo demonstration group than in the athlete group, suggesting that the balance ability varies according to the form of Taekwondo exercise.

In contrast, Kim and Kim and Shin [23] examined the isokinetic muscular function, dynamic stability, and static stability before and after muscle fatigue with 15 Kyorugi athletes and 15 Poomsae athletes. They reported a decrease in maintaining static balance for both groups, indicating no significant difference between the two groups. A comparison of the two groups in static postural stability after fatigue induced, the anterior-posterior balance was significantly higher in the Kyorugi group than the Poomsae group, whereas the medial-lateral balance increased significantly in both groups. No statistical difference in the comparison of the dynamic postural stability was observed between the groups.

The present study showed no difference in all measured variables between the two groups despite the different kinematic properties involved in each group, possibly being the result of both Kyorugi and Poomsae commonly requiring muscle strength and balance ability. In other words, the common kinematic properties involved in both forms of Taekwondo may have contributed to the lack of difference in the measured variables between the groups. In addition, adopting the aerobic fatigue-inducing method instead of the anaerobic method for muscle fatigue inducement could have been insufficient to represent the proprioception of Kyorugi athletes who are typically trained for anaerobic fitness and capacity. Therefore, future studies will be needed to complement this aspect and adopt different methods of muscle fatigue inducement.

This study had some limitations. The sample group was too small to represent the entire population of Taekwondo athletes and that it was not a direct investigation of 
proprioception and sports injuries in Taekwondo athletes. Therefore, future studies will be needed to examine how muscle fatigue directly affects proprioception, which subsequently influences the balance and kinetic variables in Taekwondo athletes. Such a study will build a knowledge base for sports injuries in Taekwondo athletes during training and competition and promote further research on providing intervention programs for the prevention of sports injuries. In addition, considering the kinematic characteristics of Kyorugi and Poomsae athletes, it will be necessary to study the changes in proprioception according to the kinetic characteristics of the two groups using different fatigueinducing methods.

\section{Conclusion}

The results from existing studies that are related directly or indirectly to this study provide sufficient evidence that muscle fatigue induced in Taekwondo athletes can lead to cause sports injuries. Understanding the degraded proprioception due to muscle fatigue can be used to determine preemptively when sports injuries and a decrease in performance due to muscle fatigue are likely to occur during competition or training. This will also contribute to injury prevention and athlete protection. In addition, the information can be used as basic data for the development of sports injuries prevention and rehabilitation programs for Taekwondo athletes.

\section{References}

[1] Kim HC, Kim YJ. A research into Injuries to Taekwondo Trainees during Exercise - Focusing on South Chung cheong. Journal of Digital Convergence Provinc. 2013; 11(4):415-30.

[2] Jung HD. A Statistical Study on Injuries occurring in High School Taekwondo Players. Journal of Korean Society for the Study of Physical Education. 2010;
15(2):295-310.

[3] Kim WK., Jeon MJ. Study on the isokinetic muscle strength and muscle endurance of male high school Taekwondo athletes, Korean, Journal of Physical Education. 2006; 45(5);381-8

[4] Manion A.F, Connolly B, Wood K, et al. The use of surface EMG power spectral analysis in the evaluation of back muscle function. J Rehail Res Dev, 1997;34: 427-39.

[5] Yu WD, Chu ZS, Jang SM, et al. The Mediating Effect of Satisfaction on the Exercise Duration and Persistence of Taekwondo Practitioners. Journal of the Korean Data Analysis Society. 2018;20(1):415-26.

[6] Choi BO, Kim MW, Hong HS. The Effects of Lower Extremity Muscular Fatigue on Proprioceptive Position Sense of the Knee Joint. Archives of Orthopedic and Sports Physical Therapy. 2009;5(1):19-26.

[7] Yang KH, Lee HO. The knee joint position sense in healthy women. PNF and Movement. 2010;8(1):31-9.

[8] Bressel E, Yonker JC, Kras J, et al. Comparison of static and dynamic balance in female collegiate soccer, basketball, and gymnastics athletes. J Athl Trai. 2007; 42(1):42-6,

[9] Kauffman KL, Nashner LM, Allison LK. Balance is a critical parameter in orthopedic rehabilitation. Orthopedic Physical Therapy Clinics of North America. 1997;6(1): 1059-516.

[10] Kim TH, Youm CH. Effects of Knee Joint Muscle Fatigue and Overweight on Shock Absorption during Single-Leg Landing of Adult Women. Korean Journal of Sport Biomechanics. 2014;24(1):59-66.

[11] Khil JH, Son KH. Influence of Long-term Taekwondo Poomsae Training on Static Balance, Dynamic Balance, and Gait Balance. The Official Journal of the Korean Association of Certified Exercise Professionals. 2012; 14(2):117-26.

[12] Jang SM, Jung YJ. The Effect of Participation Motivation and Ego-Resilience of Taekwondo Trainees on 
Psychological Well-Being. JKDAS. 2018;20(1):403-13.

[13] Erdfelder E, Faul F, Buchner A. GPOWER: A general power analysis program. Behavior Research Methods, Instruments \& Computers. 1996;28:1-11.

[14] Adlerton AK, Moritz U, Moe-Nilssen R. Forceplate and accelerometer measures for evaluating the effect of muscle fatigue on postural control during one-legged stance. Physiotherapy Research International. 2003;8(4):187-99.

[15] Froyd C, Millet GY, Noakes TD. The development of peripheral fatigue and short-term recovery during self-paced high-intensity exercise. J Physio. 2013;591(5): $1339-46$.

[16] Toby OS, Leigh Davies, Caroline BH. A Systematic Review to Determine the Reliability of Knee Joint Position Sense Assessment Measures. Knee. 2013;20(3):162-9.

[17] Seo BD, Shin HS, Kwon TY. Lower-Extremity Plyometric Training Improves Postural Stability in Young Male Athletes. Journal of The Korean Data Analysis Society. 2010;12(6):2943-52.

[18] Brent IS, Carrie LD, Janet Simon, Joanne Klossner, John Schrader. Ankle Strength and Force Sense After a Progressive, 6-week Strength-Training Program in People With Functional Ankle Instability. J Athl Train. 2012; 47(3):282-8.

[19] Faunø PI, Wulff Jakobsen B. Mechanism of anterior cruciate ligament injuries in soccer. Int J Sports Med. 2006;27(1):75-9.

[20] Lee WH, Kang TH, Kim JH. Effect of Various LegCrossing Positions on Muscle Activities of Rectus Femoris. Tensor Fascia Latae, and Hamstring in Healthy 20's Adults, The journal of Korean society of physical therapy. 2015;27(5): 315-9.

[21] Shin YH, Youm,CH. Effects of Muscle Fatigue on Ankle Joint and the Fatigue Protocols of Postural Control during Single-Leg Stance. The Korean Journal of Dance. 2013; 71(3):25-40

[22] Seo BD, Shin HS, Han DW. Comparison of the hamstring-to-quadriceps strength ratio of collegiate soccer player and age-matched healthy students. Korean Journal of Sport Biomechanics. 2009;19(4):617-25.

[23] Kim HD. Kim DH. Shin, Y. S. Differential effects of Taekwondo training on postural stability after fatigue induced by aerobic endurance exercise. Exercise Science. 2011;20(3):215-26.

[24] Cha Y, Oh JK. Difference of lower extremity, trunk muscle strength, balance ability and proprioception among Korea national Taekwondo demonstration player, competition player and trainee. The Research Institute of Physical Education \& Sports Science. 2016;33(2): 175-84. 